\title{
Unexpected High Stiffness of Ag and Au Nanoparticles
}

\author{
Q.F. Gu, G. Krauss, ${ }^{*}$ and W. Steurer \\ Laboratory of Crystallography, Department of Materials, ETH Zurich, CH-8093 Zurich, Switzerland \\ F. Gramm \\ Laboratory of Solid State Physics, EMEZ, ETH Zurich, CH-8093 Zurich, Switzerland
}

\section{A. Cervellino}

Laboratory for Neutron Scattering, ETH Zurich and PSI Villigen, CH-5232 Villigen PSI, Switzerland

(Received 30 August 2007; published 28 January 2008)

\begin{abstract}
We studied the compressibility of silver $(10 \mathrm{~nm})$ and gold $(30 \mathrm{~nm})$ nanoparticles, $\mathrm{n}-\mathrm{Ag}$ and $\mathrm{n}-\mathrm{Au}$, suspended in a methanol-ethanol mixture by $\mathrm{x}$-ray diffraction (XRD) with synchrotron radiation at pressures up to $30 \mathrm{GPa}$. Unexpectedly for that size, the nanoparticles show a significantly higher stiffness than the corresponding bulk materials. The bulk modulus of n-Au, $K_{0}=290(8) \mathrm{GPa}$, shows an increase of ca. $60 \%$ and is in the order of $\mathrm{W}$ or Ir. The structural characterization of both kinds of nanoparticles by $\mathrm{XRD}$ and high-resolution electron microscopy identified polysynthetic domain twinning and lamellar defects as the main origin for the strong decrease in compressibility.
\end{abstract}

DOI: 10.1103/PhysRevLett.100.045502

Reaching the nanoscale, mechanical properties of materials were found to persist as well as vary with size compared to the microcrystalline (bulk) materials. The elastic modulus, for example, increases for a large number of materials [1-3]. On the other hand the bulk modulus of some materials was found independent on the particle size [4]. Nanoparticles of $\mathrm{Fe}$ [5] and $\mathrm{Ni}$ [6], for example, showed the same bulk modulus as the bulk material. Structural features affecting the mechanical properties are discussed in the literature, but the interrelationships are not yet fully understood. The apparently controversial findings on the behavior of nanoparticles as a function of pressure were the motivation of the present study on $\mathrm{Ag}$ and $\mathrm{Au}$ nanoparticles (n-Ag and $\mathrm{n}-\mathrm{Au}$ ).

Silver and gold crystallize with the face-centered cubic (fcc) structure and have been thoroughly studied theoretically and experimentally. The compressibility of bulk $\mathrm{Ag}$ was investigated up to $150 \mathrm{GPa}$ [7] without any indications for a high-pressure modification. Bulk Au was also studied by compressibility measurements up to high pressures, e.g., [8]. Recently, Dubrovinsky et al. [9] observed a transition to a hexagonal close-packed structure above $240 \mathrm{GPa}$. To the best of our knowledge, only Au and no Ag nanoparticles were studied in situ at high pressures up to now. Singh et al. [10] performed nonhydrostatic compression experiments on gold powder and found a higher strength of the n-Au compared to the bulk gold. Martin et al. [11] measured the pair distribution function (PDF) of gold nanoparticles up to $10 \mathrm{GPa}$ and found a slightly smaller $\mathrm{Au}-\mathrm{Au}$ distance from $\mathrm{PDF}$ than from Rietveld refinements.

The investigated spherical nanoparticles were commercial Ag (99.9\% pure, $10 \mathrm{~nm}$ mean particle size) and $\mathrm{Au}$ (99.9\% pure, $30 \mathrm{~nm}$ ), both from Nanostructured \& Amorphous Materials, Inc., USA. The microstructural
PACS numbers: 62.50.- $\mathrm{p}$, 61.46.Df, 61.66.Bi, 62.25. $-\mathrm{g}$

analysis of the particles was done using a Philips CM30 high-resolution transmission electron microscope (HRTEM). The lattice parameters were determined on a diffractometer consisting of a Huber Eulerian cradle and a Marresearch mar345 image-plate scanner using $\mathrm{Ag} K \alpha$ radiation and silicon (NBS640a) as internal standard. The 2D diffraction images were integrated by the use of the program fit2d [12] and the 1D powder pattern were refined by applying the Rietveld-method using the program GSAS $[13,14]$. Synchrotron x-ray diffraction experiments were performed at the Materials Science beam line, Swiss Light Source (SLS) at Paul Scherrer Institut (PSI, Villigen, Switzerland). 2D diffraction data was collected on a Marresearch mar345 image-plate detector using a wavelength of $\lambda=0.5640 \AA$. The data evaluation was done as described above. High pressures of up to 27.8(1.1) GPa for $\mathrm{n}-\mathrm{Ag}$ and 30.6(8) GPa for n-Au were generated by use of an ETH-type diamond anvil cell. A mixture of methanol and ethanol (4:1) served as pressure-transmitting medium. To minimize deviatoric stress, the amount of pressure medium covered ca. 30\% of the total volume (nanoparticles 70\%) of the sample chamber within the stainless steel gasket. A ruby crystal was placed into the sample chamber as a pressure marker and the pressures were determined using the ruby fluorescence technique. Equations of state were calculated by the use of the program EosFit5.2 [15].

The size and microstructure of the particles was characterized by HRTEM. A qualitative EDX analysis of the particles showed only $\mathrm{Ag}$ and $\mathrm{Au}$, respectively, as detectable elements. Figure 1 shows typical images of the spherical particles. In the high-resolution images, several types of twinning are observable. These kinds of twinning are typical for Au nanoparticles and described in detail, for example, in [16-18] as multiply twinned particles (MTP). Comprehensive twinning models were derived by Yang 


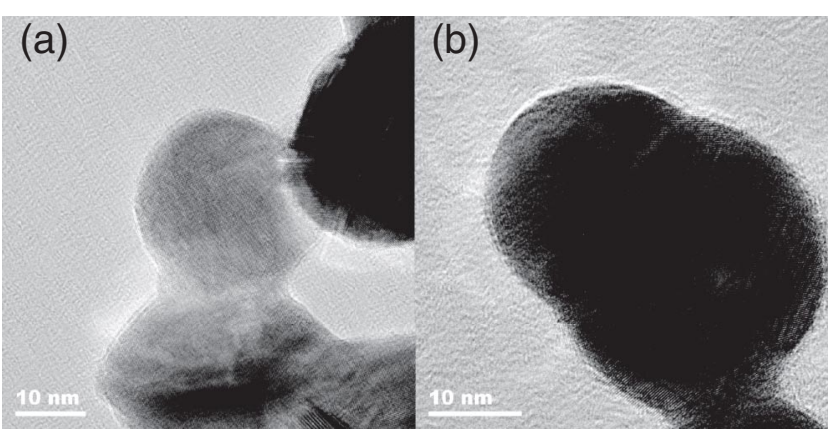

FIG. 1. HRTEM images of n-Ag (a) and n-Au (b) showing the typical twinned microstructures. Note that n-Ag particles already started to melt in the microscope.

[19]. For the Ag particles, we observed a melting during the HRTEM investigations as reported for Au nanoparticles [17]. The lattice parameters were determined by xray diffraction using silicon as an internal standard. The obtained values of 4.081(1) $\AA$ for $\mathrm{n}-\mathrm{Ag}$ and 4.076(1) $\AA$ for $\mathrm{n}$ - $\mathrm{Au}$ are smaller than for the respective bulk metals (4.08626(4) $\AA$ for $\mathrm{Ag}$ and 4.07894(5) $\AA$ for $\mathrm{Au}[20]$ ), caused by strain induced by the twin boundaries and the surface. The deviation of the lattice parameter of $\mathrm{n}-\mathrm{Ag}$ is much larger than of $\mathrm{n}-\mathrm{Au}$, which is reasonable due to the smaller particle size (10 and $30 \mathrm{~nm}$, respectively). An increase of the lattice parameter as described by Shyjumon et al. [21] for Ag nanoparticles deposited on a $\mathrm{Si}$ surface was not observed. During the lattice parameter refinements an obvious discrepancy in the intensity ratio of the 111 and 200 reflections was observed. Based on the simple fcc-model, the pattern could not be refined successfully by introducing preferred orientation or strain. Therefore more complex real structure models affecting intensity and peak broadening were applied in a Rietveldlike whole pattern refinement procedure [22,23] (Fig. 2). The method is based on three cluster models with fivefold symmetry $[16,24]$, which are built from $\{111\}$-tetrahedra with fcc-structure sharing a common edge as (pseudo) fivefold axis in lattice direction [101]]: cluster type $A$ (pentagonal): five truncated tetrahedra deformed to build an ideal pentagonal cluster; cluster type B (five fcc incoherent domains): five undeformed truncated tetrahedra with five small wedge gaps; cluster type $C$ (multitwinned): five undeformed truncated tetrahedra with "perfect twinning" and only one wedge gap. The refinement procedure yields the number fraction of each cluster type. According to the refinement, $\mathrm{n}$-Au consists mainly of cluster type $\mathrm{B}$ while $\mathrm{n}-\mathrm{Ag}$ consists mainly of cluster type A (Table I) [24]. Compared to the obtained HRTEM images, the microstructural description of the particles based on only three idealized models is a strong simplification but reflects the kind and high amount of structural disorder mainly caused by twin boundaries within the nanoparticles.

XRD pattern of $\mathrm{n}-\mathrm{Ag}$ and $\mathrm{n}-\mathrm{Au}$ were collected up to a pressure of ca. $30 \mathrm{GPa}$. No phase transformation or non-

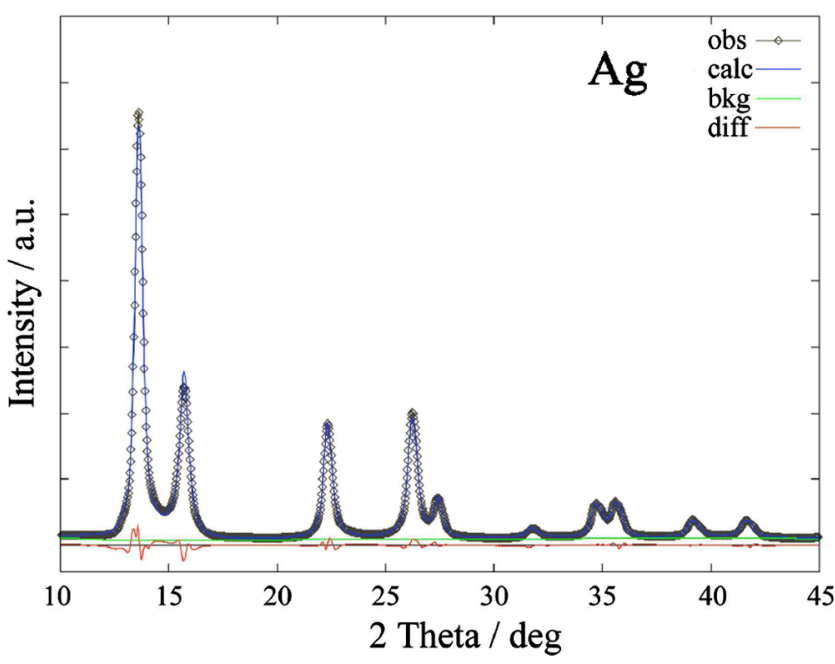

FIG. 2 (color online). Rietveld-like refinement of the diffraction pattern of $\mathrm{n}-\mathrm{Ag}$ at ambient pressure based on the cluster models $\mathrm{A}, \mathrm{B}$, and $\mathrm{C}$ as described in the text. The comparable result of the refinement for $\mathrm{n}-\mathrm{Au}$ is shown in [24], respectively.

cubic lattice distortions were observed in the investigated pressure range. With the described setup, diffraction patterns covering the $111,200,220,311$, and 222 reflections could be measured at each pressure. The $d$-values were determined by fitting a pseudo-Voigt function to the diffraction profiles. The lattice parameters of $n-A g$ and $n-A u$ at each pressure were averaged from the values calculated for each of the $d$ values of the five observed reflections listed above [24]. Figure 3 shows the fit of the experimental volume to a 3rd-order Birch-Murnaghan (BM3) equation of state (EOS) for the pressure $P=3 K_{0} f_{E}\left(1+2 f_{E}\right)^{5 / 2} \times$ $\left[1+\frac{3}{2}\left(K^{\prime}-4\right) f_{E}\right]$, with $f_{E}=\left[\left(V_{0} / V\right)^{2 / 3}-1\right] / 2$ the Eulerian strain, $K_{0}$ the bulk modulus, $K^{\prime}$ its pressure derivative, $V$ and $V_{0}$ the unit cell volume and volume at zero pressure. Unexpectedly for the size of the particles, the compressibility is much lower than for the bulk material. This behavior is present below $10 \mathrm{GPa}$, the maximum pressure governing hydrostatic conditions with the present setup [25], as well as above that pressure. The obtained values of the bulk modulus at zero pressure are given in Table II together with literature data. The results based on 2nd- and 3rd-order BM EOS are also given. For n-Au the differences of the obtained values are within the errors. For $\mathrm{n}$-Ag the differences are significant. A large value of $K^{\prime}$ was obtained based on the 3rd-order fit, uncommon to bulk metals and a bulk modulus comparable to bulk Ag. Nevertheless the curve for bulk Ag is well below the curve

TABLE I. Amounts (number fractions) of clusters A, B, and C obtained from a Rietveld-like refinement of the XRD patterns.

\begin{tabular}{lccc}
\hline \hline & cluster A & cluster B & cluster C \\
\hline n-Ag & $44.1 \%$ & $19.5 \%$ & $36.4 \%$ \\
n- $\mathrm{Au}$ & $24.7 \%$ & $45.1 \%$ & $30.2 \%$ \\
\hline \hline
\end{tabular}




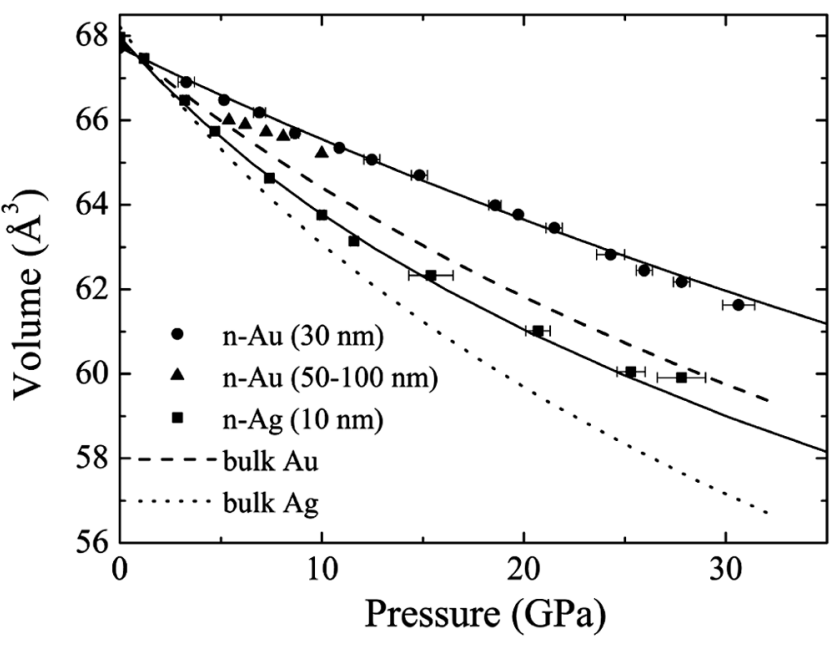

FIG. 3. Pressure dependence of the unit cell volume of less compressible $\mathrm{n}-\mathrm{Ag}$ and $\mathrm{n}-\mathrm{Au}$ compared to the bulk materials. The data for $\mathrm{n}-\mathrm{Au}(50-100 \mathrm{~nm})$ is taken from [11]. The solid lines correspond to fits of BM3 EOS. Dotted and dashed lines represent BM3 EOS for bulk Ag [26] and Au [29], respectively. Errors in volumes are in the size of the symbols.

for $\mathrm{n}-\mathrm{Ag}$ indicating the higher stiffness of the $\mathrm{n}-\mathrm{Ag}$. The different behavior of $\mathrm{n}-\mathrm{Ag}$ and $\mathrm{n}-\mathrm{Au}$ could be explained by the higher compressibility of $\mathrm{n}-\mathrm{Ag}$, which makes effects of the nonhydrostatic pressure medium to become more prominent.

As the particles are quite large, effects caused by a large surface to bulk ratio are assumed not to play a significant role. In Fig. 3 results of a PDF-study on larger Au nanoparticles $(50-100 \mathrm{~nm})$ is included [11]. Based on the lattice parameters given in their paper, the bulk modulus was calculated as 210(14) GPa, which is lower than the value obtained in this study, but still higher than the value for bulk $\mathrm{Au}$. For these even larger nanoparticles, surface effects can be ruled out. But how can the increase be understood? Using the relation derived by Einstein [30], the bulk modulus $K_{0}$ is correlated to the interatomic distances $d_{A-A}$

TABLE II. EOS data for nanocrystalline and bulk Ag and Au. The value for $50-100 \mathrm{~nm} \mathrm{n}$-Au was calculated based on lattice parameters from [11].

\begin{tabular}{lcllllc}
\hline \hline & size/(nm) & EOS & $V_{0} /\left(\AA^{3}\right)$ & $K_{0} /(\mathrm{GPa})$ & \multicolumn{1}{c}{$K^{\prime}$} & Ref. \\
\hline $\mathrm{n}-\mathrm{Ag}$ & 10 & BM2 & $67.97(2)$ & $139(3)$ & 4 & $\mathrm{a}$ \\
$\mathrm{n}-\mathrm{Ag}$ & 10 & BM3 & $67.97(2)$ & $121(6)$ & $8.3(1.4)$ & $\mathrm{a}$ \\
$\mathrm{Ag}$ & bulk & $\mathrm{BM} 3$ & & 116 & 4.7 & {$[26]$} \\
$\mathrm{Ag}$ & bulk & BM3 & & 116.31 & 5.86 & {$[27]$} \\
$\mathrm{Ag}$ & bulk & Vinet & & $98(1.6)$ & $5.47(2)$ & {$[7]$} \\
$\mathrm{n}-\mathrm{Au}$ & 30 & $\mathrm{BM} 2$ & $67.71(1)$ & $286(2)$ & 4 & $\mathrm{a}$ \\
$\mathrm{n}-\mathrm{Au}$ & 30 & $\mathrm{BM} 3$ & $67.71(1)$ & $290(8)$ & $3.4(9)$ & $\mathrm{a}$ \\
$\mathrm{n}-\mathrm{Au}$ & $50-100$ & $\mathrm{BM} 2$ & $67.8(1)$ & $210(14)$ & 4 & {$[11]$} \\
$\mathrm{Au}$ & bulk & $\mathrm{BM} 3$ & & 171 & 5 & {$[28]$} \\
$\mathrm{Au}$ & bulk & $\mathrm{BM} 3$ & & $167(11)$ & $5.5(8)$ & {$[29]$} \\
$\mathrm{Au}$ & bulk & BM3 & & $172.5(1.4)$ & $5.4(8)$ & {$[8]$} \\
\hline \hline
\end{tabular}

${ }^{\mathrm{a}}$ This study; for details see text. by

$$
K_{0} \propto \frac{M \theta_{E}^{2}}{d_{A-A}}
$$

with $M$ the molar mass and $\theta_{E}$ the Einstein temperature. For nanoparticles, the Einstein temperature can be particularly determined from the mean square relative displacement parameters obtained by temperature dependent extended x-ray absorption fine structure (EXAFS) measurements with very high signal-to-noise ratios [31,32]. Recently, a study on $\mathrm{ZnS}$ nanoparticles showed an increase of the Einstein temperature with decreasing particle size [33] which should imply structural stiffening. A highpressure study on these particles unfortunately did not confirm an increased stiffness [34]. The authors claimed additional structural responses like bond torsion as a possible reason for the decrease. An increase of the Einstein temperature was also found for Ge nanoparticles embedded in silica [35], whereas Ag nanoparticles embedded in silicate glass showed a small decrease of the Einstein temperature [36]. It has to be noted that the matrix has a strong effect on the properties of the nanoparticles. In contrast to $\mathrm{ZnS}$ with a high amount of directed covalent bonding, $\mathrm{Ag}$ and $\mathrm{Au}$ have no directional bonds, i.e., bond torsion or bond bending can be ruled out as pressure compensating mechanisms. For free Ag or Au nanoparticles unfortunately no temperature dependent studies determining the Einstein temperatures are present to the literature (to the best of our knowlegde). Based on the findings of Gilbert et al. [33] and Araujo et al. [35] the present results can be explained as follows: The smaller lattice parameter of $\mathrm{n}-\mathrm{Ag}$ and $\mathrm{n}-\mathrm{Au}$ clearly indicates strain induced by the twin boundaries and the surface, which may be present also in larger nanoparticles [37]. The strain leads to shorter interatomic distances, i.e., bond length compression within the particles and this leads to an increase of the bulk modulus [Eq. (1)]. An increase in the Einstein temperature will also increase the bulk modulus. Gilbert et al. [33] explained the increase in the Einstein temperature of $\mathrm{ZnS}$ nanoparticles by the highly disordered structure. As the HRTEM studies show, the microstructures of the investigated Ag and Au nanoparticles are also highly disordered, caused by coherent and incoherent twin domains. Therefore, it may be assumed, that the Einstein temperature is also increased for these nanoparticles. The bulk modulus is related to the square of the Einstein temperature, so a small change will strongly influence the property. As the results of the refinements indicate, nAg contains a higher amount of coherently twinned particles than $\mathrm{n}-\mathrm{Au}$ (Table I). As a consequence, $\mathrm{n}-\mathrm{Au}$ has a broader distribution of bond lengths than $\mathrm{n}-\mathrm{Ag}$. This could be the reason for the much higher increase of the bulk modulus of $\mathrm{n}-\mathrm{Au}$ than of $\mathrm{n}-\mathrm{Ag}$ as all the deviations from equilibrium bond length may increase the Einstein temperature [33]. A conceivable model for the twin boundaries could contain icosahedral clusters of atoms embedded in 
the fcc packing. Because of the higher packing density, the icosahedra are less compressible and as a packing of 20 slightly distorted tetrahedra they can be embedded with minor structural distortions into the fcc-lattice. The resulting microstructure can be described as a nanocomposite consisting of a stiffer phase (icosahedra) embedded in a softer matrix (fcc). The bulk properties would then be inbetween the values of the icosahedra and the matrix. Another reason for the bigger increase in bulk modulus of $\mathrm{n}-\mathrm{Au}$ compared to $\mathrm{n}-\mathrm{Ag}$ may be found in the bigger molecular mass, which is nearly twice as high for $\mathrm{Au}$ as for Ag. Changes in the Einstein temperature therefore will have a relatively bigger influence on the bulk modulus, respectively, [Eq. (1)]. As a conclusion, the observed trend can be understood qualitatively based on Eq. (1). For a quantitative analysis, the full phonon dispersion should be taken into account. Recently, Biener et al. [38] found an increase of yield strength with decreasing sample size of nanopourous gold, which is in accordance with our findings.

In summary, it was shown that the investigated nanoparticles of $\mathrm{Ag}$ and $\mathrm{Au}$ are significantly stiffer than the corresponding bulk materials. This behavior may be explained by the disordered microstructure of the particles. To confirm the explanation given in this Letter, temperature dependent EXAFS studies should be performed to determine the Einstein temperatures of $\mathrm{n}-\mathrm{Ag}$ and $\mathrm{n}-\mathrm{Au}$ and obtain a closer insight to the microstructure of the particles. The very recently reported availability of nanoparticles with defined crystallinity [39] offers an excellent basis to answer these fundamental questions. The unexpected high stiffness may open new fields of applications to these noble metal nanoparticles and it could be expected that other metal nanoparticles show a similar trend.

Part of this work was performed at the Swiss Light Source, Paul Scherrer Institut, Villigen, Switzerland. We thank the staff of the MS beam line for experimental assistance. This work was supported by the Swiss National Science Foundation (SNF) under Grant No. 200020-115871.

*guenter.krauss@mat.ethz.ch

[1] S. H. Tolbert and A.P. Alivisatos, Science 265, 373 (1994).

[2] J.Z. Jiang, J.S. Olsen, L. Gerward, and S. Morup, Europhys. Lett. 44, 620 (1998).

[3] Z. Wang, Y. Zhao, D. Schiferl, J. Qian, R. T. Downs, H.-K. Mao, and T. Sekine, J. Phys. Chem. 107, 14151 (2003).

[4] S. H. Tolbert and A. P. Alivisatos, Annu. Rev. Phys. Chem. 46, 595 (1995).

[5] B. Chen, D. Penwell, M. B. Kruger, A.F. Yue, and B. Fultz, J. Appl. Phys. 89, 4794 (2001).

[6] S. Rekhi, S. K. Saxena, R. Ahuja, B. Johansson, and J. Hu, J. Mater. Sci. 36, 4719 (2001).

[7] Y. Akahama, H. Kawamura, and A. K. Singh, J. Appl. Phys. 95, 4767 (2004).
[8] A. Dewaele, P. Loubeyre, and M. Mezouar, Phys. Rev. B 70, 094112 (2004).

[9] L. Dubrovinsky, N. Dubrovinskaia, W. A. Crichton, A. S. Mikhaylushkin, S. I. Simak, I. A. Abrikosov, J.S. de Almeida, R. Ahuja, W. Luo, and B. Johansson, Phys. Rev. Lett. 98, 045503 (2007).

[10] A. K. Singh, H. P. Liermann, S. K. Saxena, H. K. Mao, and S. Usha Devi, J. Phys. Condens. Matter 18, S969 (2006).

[11] C. D. Martin, S. M. Antao, P. J. Chupas, P. L. Lee, S. S. Shastri, and J. B. Parise, Appl. Phys. Lett. 86, 061910 (2005).

[12] A. P. Hammersley, S. O. Svensson M. Hanfland, A. N. Fitch, and D. Häussermann, High Press. Res. 14, 235 (1996).

[13] A. C. Larson and R.B. Von Dreele, General Structure Analysis System (GSAS), Los Alamos National Laboratory Report, laur 86-748 ed. 2000.

[14] B. H. Toby, J. Appl. Crystallogr. 34, 210 (2001).

[15] R. J. Angel, Rev. Mineral. Geochem. 41, 35 (2000).

[16] S. Ino, J. Phys. Soc. Jpn. 21, 346 (1966).

[17] P. Buffat, Mater. Chem. Phys. 81, 368 (2003).

[18] K. Koga and K. Sugawara, Surf. Sci. 529, 23 (2003).

[19] C. Y. Yang, J. Cryst. Growth 47, 274 (1979).

[20] M.E. Straumanis, Monatsh. Chem. 102, 1377 (1971).

[21] I. Shyjumon, M. Gopinadhan, O. Ivanova, M. Quaas, H. Wulff, C. A. Helm, and R. Hippler, Eur. Phys. J. D 37, 409 (2006).

[22] A. Cervellino, C. Giannini, and A. Guagliardi, J. Appl. Crystallogr. 36, 1148 (2003).

[23] A. Cervellino, C. Giannini, A. Guagliardi, and D. Zanchet, Eur. Phys. J. B 41, 485 (2004).

[24] See EPAPS Document No. E-PRLTAO-100-069803 for supplementary materials. For more information on EPAPS see http://www.aip.org/pubservs/epaps.html.

[25] R. J. Angel, M. Bujak, J. Zhao, G. D. Gatta, and S. D. Jacobsen, J. Appl. Crystallogr. 40, 26 (2007).

[26] K. Syassen and W. B. Holzapfel, J. Appl. Phys. 49, 4427 (1978).

[27] J. J. Xie, S. P. Chen, S. de Gironcoli, and S. Baroni, Philos. Mag. B 79, 911 (1999).

[28] K. Takemura, J. Appl. Phys. 89, 662 (2001).

[29] D. L. Heinz and R. Jeanloz, J. Appl. Phys. 55, 885 (1984).

[30] A. Einstein, Ann. Phys. (Leipzig) 34, 170 (1911).

[31] G. Dalba and P. Fornasini, J. Synchrotron Radiat. 4, 243 (1997).

[32] A. I. Frenkel and J. J. Rehr, Phys. Rev. B 48, 585 (1993).

[33] B. Gilbert, F. Huang, H. Zhang, G. A. Waychunas, and J. F. Banfield, Science 305, 651 (2004).

[34] B. Gilbert, H. Zhang, B. Chen, M. Kunz, F. Huang, and J. F. Banfield, Phys. Rev. B 74, 115405 (2006).

[35] L. L. Araujo, P. Kluth, G. de M. Azevedo, and M.C. Ridgway, Phys. Rev. B 74, 184102 (2006).

[36] M. Dubiel, S. Brunsch, and L. Tröger, J. Phys. Condens. Matter 12, 4775 (2000).

[37] G. Ouyang, X. L. Li, X. Tan, and G. W. Yang, Appl. Phys. Lett. 89, 031904 (2006).

[38] J. Biener, A. M. Hodge, J. R. Hayes, C. A. Volkert, L. A. Zepeda-Ruiz, A. V. Hamza, and F. F. Abraham, Nano Lett. 6, 2379 (2006).

[39] Y. Tang and M. Ouyang, Nat. Mater. 6, 754 (2007). 